\title{
Examining Supernova Remnant GSH054-00+003
}

\author{
Cheuk Man Lo, Dr. Shauna Sallmen \\ Physics Department, University of Wisconsin- La Crosse
}

Supernova remnants play a key role in galactic evolution. Therefore, studying them intensively can improve our understanding of the process. In this project, we studied the neutral hydrogen shell GSH054-00+003 located at RA $292.75^{\circ}$ and DEC $18.25^{\circ}$ that was found in the SETHI dataset. We found its reference velocity to be $3 \mathrm{~km} / \mathrm{s}$. After matching it with the Galactic rotation curve and spiral structure, we found its distance to be $10 \mathrm{kpc}$ away. Based on this and geometry, we estimated its size to be $245 \mathrm{pc}$. Using computer analysis of its HI spectrum, we determined the expansion velocity to be $8.6 \mathrm{~km} / \mathrm{s}$. We therefore can calculate its age and kinetic energy. Assuming the number density of the ambient interstellar medium to be 1 atom $/ \mathrm{cm}^{3}$, we calculated its energy to be $1.9 \times 10^{42} \mathrm{I}$. Through comparison to computer models of shell evolution, we estimated it was formed by approximately 5 to 10 supernovae. A simple age estimation is 14 million years. Although this is an over estimate, the slow expansion and absence of x-ray emission indicate the shell is old. We conclude that a cluster of supernovae formed this old shell.

\section{Introduction}

The Interstellar Medium (ISM) is the dust and gas between stars in galaxies. It is filled with shells and bubbles blown by winds from stars and supernova explosions of dying stars. Following these events, the hot star material expands outward forming a shell. It eventually cools, slows down and mixes with the ISM.

Originally, the universe was filled with only hydrogen and helium. Heavier elements such as oxygen and iron (necessary for human life) are made by fusion in stars. Elements heavier than iron are made during supernova explosions. These materials eventually distribute throughout the ISM as shells expand and merge with their surroundings. Ultimately, these new elements add to the ingredients for new stars and planetary systems: that is the reason why we can exist today. Since shell material is included in the formation of new stars and planets, it is important to study interstellar shells to understand the ecology of our Milky Way Galaxy. Although scientists understand the big picture of how shells evolve, there are details that we have yet to explain (eg. see review by Cox 2005). Intensive study of the size, age and energy of specific shells can help us to understand their features and evolution processes better. 
Cold neutral hydrogen (HI) emits light with a wavelength of $21 \mathrm{~cm}$, visible with radio telescopes. As supernova remnants look like shells and bubbles, we can search for them in this wavelength as they cool down. In this project, we used the SETHI (Search for Extra-Terrestrial HI) dataset (Korpela et al. 2002, 2004) to examine shell GSH054-00+003. This survey contains images of patches of the sky showing gas moving at different radial velocities. For every velocity slice, it contains $21-\mathrm{cm}$ emission from $\mathrm{HI}$ gas, averaged over $1.5 \mathrm{~km} / \mathrm{s}$ in radial velocity. The Galactic coordinates and radial velocity of a shell determine its name. For the shell GSH054-00+003 that we studied, it is located at Galactic longitude $+53.6^{\circ}$ and latitude $-0.1^{\circ}$. The reference velocity is $3 \mathrm{~km} / \mathrm{s}$, relative to the Local Standard of Rest. Using the SETHI data, we found the shape, size, location, and expansion velocity of this shell. We then estimated its age and kinetic energy enabling us to explore its history and evolution.

\section{Methods}

In this project, we used the computer program kvis (Gooch, 1996) to examine the SETHI shell images at the $21 \mathrm{~cm}$ wavelength, and IDL (Interactive Data Language) to analyze the shell's properties. We studied shell GSH054-00+003, which was originally found in the SETHI survey (Sallmen et al., in prep). It is located at right ascension (RA) $19 \mathrm{~h} 31 \mathrm{~m}$ and declination (DEC) $18.25^{\circ}$. We determined the location, mean angular diameter and the reference velocity by examining the SETHI images. The shape of the shell is elliptical. Therefore, we could not measure its diameter directly. We measured the length of the major and minor axes of the shell and averaged them to approximate its mean angular diameter. Figure 1 shows GSH054-00+003 at velocity $3.18 \mathrm{~km} / \mathrm{s}$. Observe that there is a black stripe, resulting from dense $\mathrm{HI}$ in the spiral arm. This shell was found near the spiral arm. 


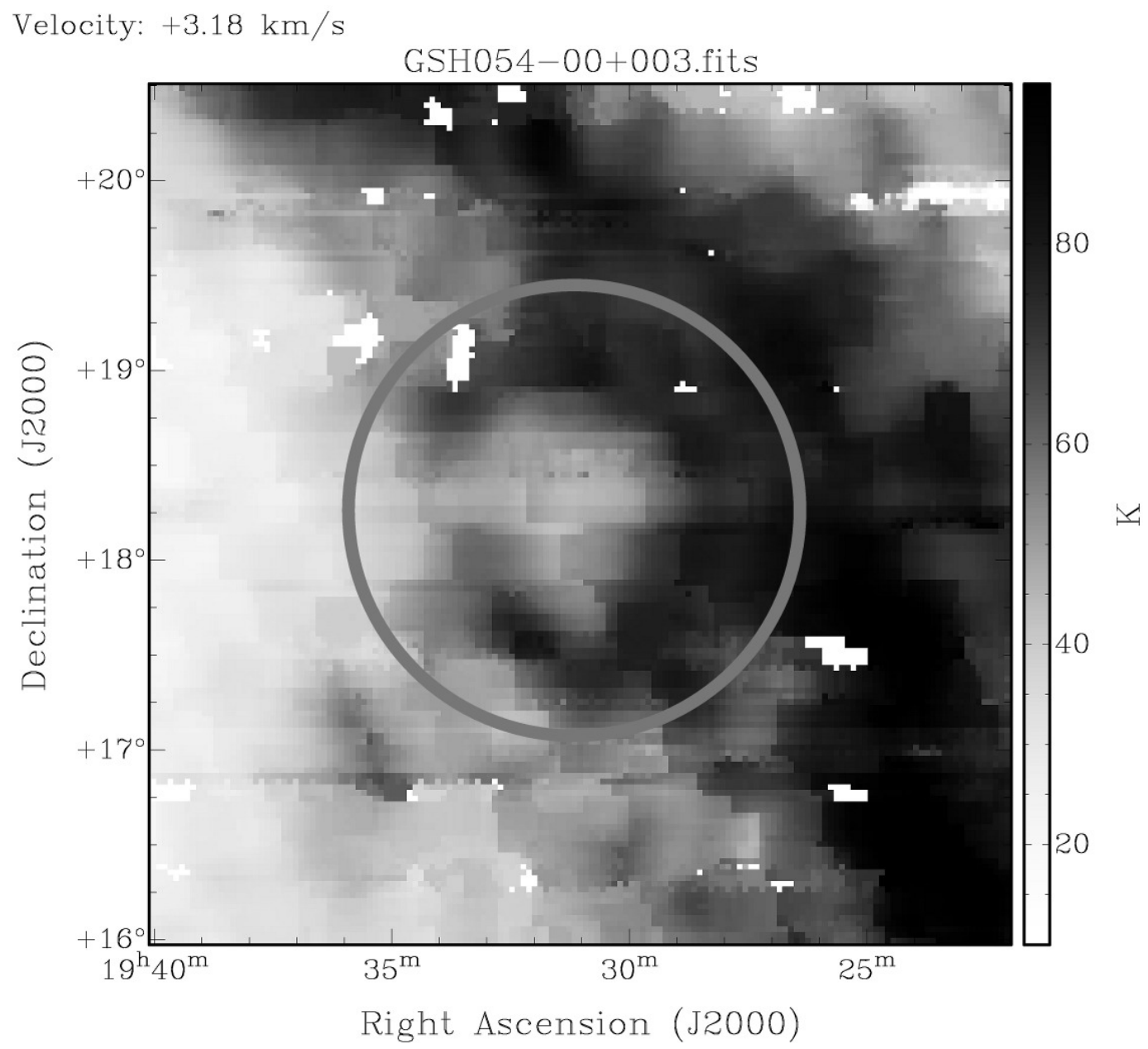

Fig.1 21-cm image of GSH054-00+003 at radial velocity $3.18 \mathrm{~km} / \mathrm{s}$. Black areas represent stronger $21 \mathrm{~cm}$ emission. The shell is indicated by the light grey circle.

Then we used the reference radial velocity to match the rotational velocity of the Galaxy to determine the distance. As the Galaxy is rotating, objects that are in different locations in the Galaxy move at specific speeds. We therefore matched the velocity of the shell with those specific locations that share the same radial velocity in the Galaxy. Figure 2 shows the two matching distances, based on the rotation curve of Brand and Blitz (1993). One is $0.05 \mathrm{kpc}$ away and the other one is $10 \mathrm{kpc}$ away. Using the fact that the shell is near the spiral arm, we determined its distance is $10 \mathrm{kpc}$ away after matching with a map of Galactic spiral arms (Cordes and Lazio, 2003). The $10.0 \mathrm{kpc}$ distance has a lower bound of $9.65 \mathrm{kpc}$ and an upper bound of $10.43 \mathrm{kpc}$. For the $0.05 \mathrm{kpc}$ distance case, the lower bound is unconstrained and its upper bound is $0.43 \mathrm{kpc}$. The range of distance is determined using the fact that the shell is visible in 6 velocity frames (spanning $7.76 \mathrm{~km} / \mathrm{s}$ ) of the SETHI data. 


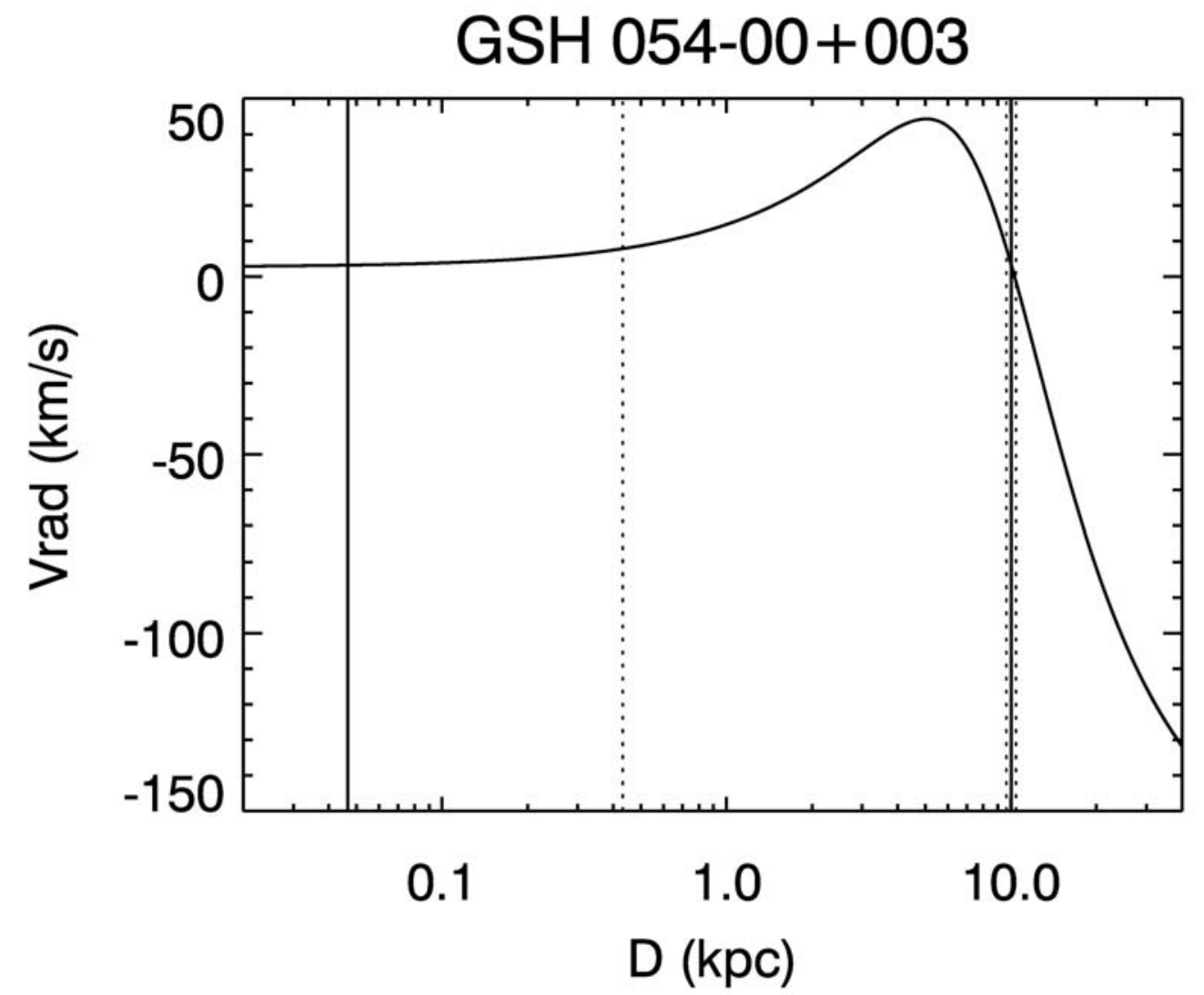

Fig.2 The solid curve represents the radial velocity of the gas along the direction RA $19 \mathrm{~h} 31 \mathrm{~m}$ and DEC $18.25^{\circ}$, according to the Galactic rotation curve (Brand and Blitz, 1993). The vertical solid lines at $10.04 \mathrm{kpc}$ and $0.05 \mathrm{kpc}$ represent distances at which the shell velocity matches the Galactic rotation curve. The dotted lines represent the error bounds.

After we found the shell's distance, we used geometry to calculate its size. Since we know the mean angular diameter of the shell, the size was calculated as: $s=\mathrm{d} \times \boldsymbol{\theta}$, where $\mathrm{d}$ is distance, $\theta$ is the mean angular diameter (in radians) and $\mathrm{S}$ is the size (diameter). To find the expansion velocity, we extracted a spectrum through the center of the shell. We also chose pixels outside the shell at the same Galactic latitude to generate a background spectrum. Figure 3 shows the results for one choice of central and background pixels. In Fig. 3, the solid line is the spectrum at the center of the shell with background subtraction. The dotted line is the spectrum without background subtraction. The dashed line is the spectrum from the surrounding background without any part of the shell. The shell's expansion signature is revealed in the local minimum and surrounding peaks near $3 \mathrm{~km} / \mathrm{s}$. 


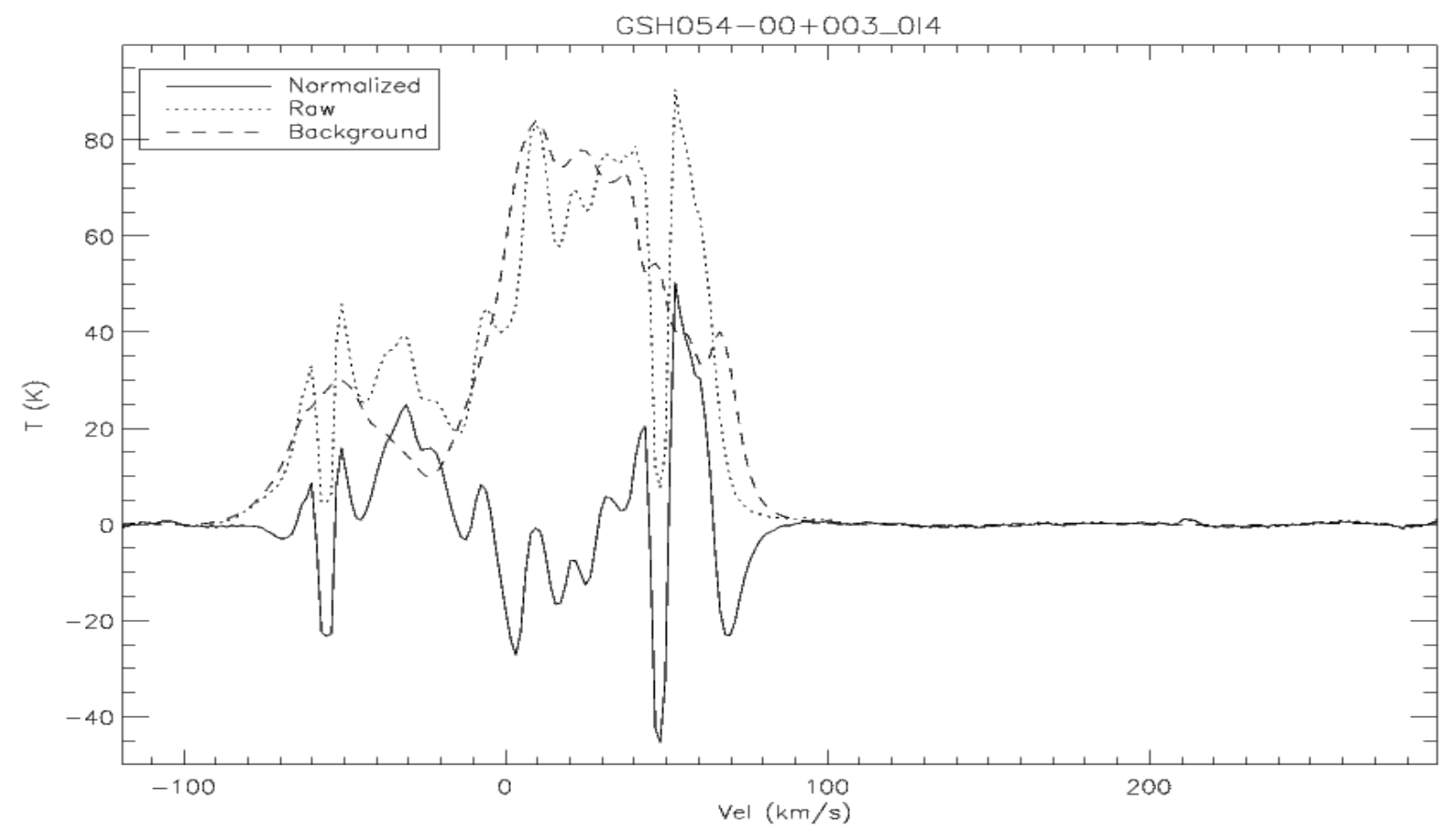

Fig 3. An example of a generated spectrum for GSH053-00+003 (21-cm emission vs. radial velocity). The solid line represents the background reduced spectrum at the center of GSH054-00+003. The dotted line represents the raw spectrum at the center of GSH054-00+003. The dashed line represents the background spectrum. The shell is at about $-10 \mathrm{~km} / \mathrm{s}$ to $10 \mathrm{~km} / \mathrm{s}$.

The background subtracted spectrum was analyzed to determine the expansion velocity as follows. The computer identified the local maxima near the reference radial velocity and set them to be the foreground wall and background wall. Figure 4 is the zoomed in background reduced spectrum. It shows that the computer found the background wall (the wall of the shell that is expanding away from us) at $+9.37 \mathrm{~km} / \mathrm{s}$ and foreground wall (the wall of the shell that is expanding toward us) at $-7.65 \mathrm{~km} / \mathrm{s}$. We also generated several more spectra using different sets of pixels. For example, we changed the size of the center point, or picked background pixels closer to or farther from the shell. Most of the choices gave results that were consistent with one another. We also compared the results to the shell images at various velocities, to ensure that the background and foreground walls were correctly identified, and not affected by noise in the data or confusion by unrelated gas. 


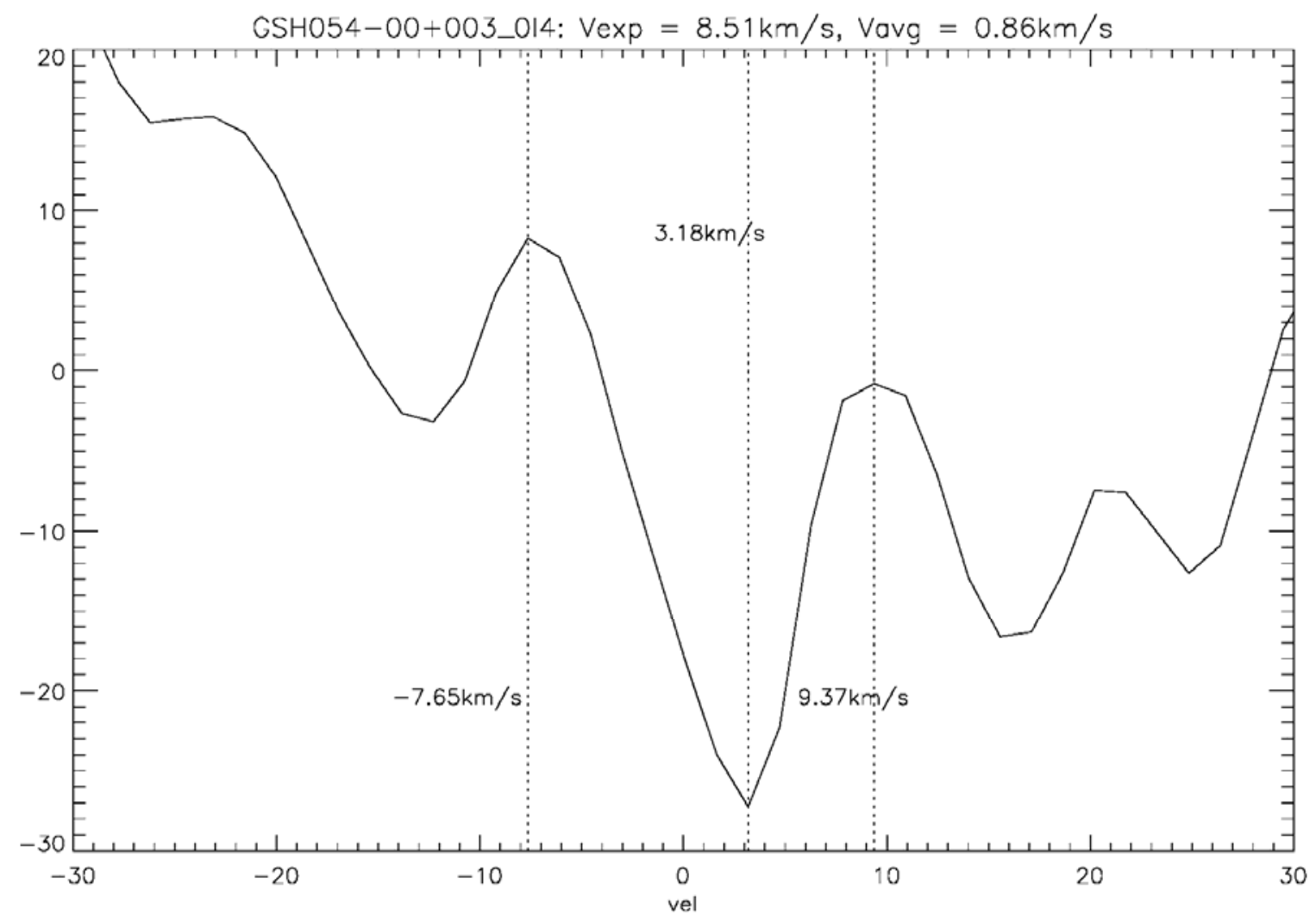

Fig 4. A zoomed in background reduced spectrum of GSH054-00+003. The solid curve represents the background subtracted HI spectrum through the center of GSH054-00+003. The dotted vertical line at $3.18 \mathrm{~km} / \mathrm{s}$ represents the radial velocity of the minimum. The dotted vertical line at $-7.65 \mathrm{~km} / \mathrm{s}$ indicates the foreground wall and the one at $9.37 \mathrm{~km} / \mathrm{s}$ indicates the background wall.

With the velocities of the foreground and background walls, the expansion velocity could then be calculated: $V_{\mathrm{exp}}=\frac{\mathrm{v}_{\mathrm{bg}}-v_{\mathrm{fr}}}{2}$. Using the expansion velocity of $8.6 \mathrm{~km} / \mathrm{s}$, we calculated the shell's age and kinetic energy and compared it to models of shell evolution. To find the age we determined how much time it took to expand to its present size: $t=\frac{d}{v_{\mathrm{w}} \mathrm{g}}$. The result of 14 million years will be an overestimate, because the shell was expanding faster early in its development.

We also wanted to estimate the kinetic energy of the shell. Therefore, we needed both the expansion velocity of the shell and its mass. To find the mass, we assumed that the shell has swept up all the material that was once inside it. The mass $m$ will then be volume times density of ambient material: $m=\frac{4}{8} \pi r^{2} \times \rho$, assuming a spherical shell. The mass density of the ambient

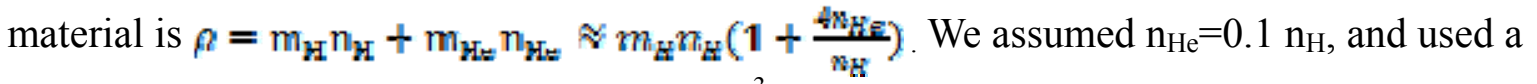
hydrogen number density $\left(\mathrm{n}_{\mathrm{H}}\right)$ of 1 atom $\mathrm{cm}^{-3}$ to calculate the mass of the shell, which was $2.7 \square 10^{5} \mathrm{M}_{\text {sun. }}$. We later also tried $10 \mathrm{~cm}^{-3}$. After finding the mass, we calculated the kinetic energy of the shell: $\mathrm{KE}=\frac{1}{2} \mathrm{~m} V_{\exp }{ }^{2}$. 


\section{Results:}

Table 1 summarizes the information on GSH054-00+003 we gathered and calculated. GSH054$00+003$ is located at RA $292.75^{\circ}$ and DEC $18.25^{\circ}$ with a mean angular diameter of $1.4^{\circ}$. It is $10.0 \pm 0.4 \mathrm{kpc}$ away from us. Using this distance and its bounds, we calculated the size to be $245 \pm 10 \mathrm{kpc}$. The shell is moving away from us with radial velocity $2 \pm 1 \mathrm{~km} / \mathrm{s}$, and expanding at $8.6 \pm 1.5 \mathrm{~km} / \mathrm{s}$. The error of the expansion velocity is determined by the resolution of the images. Based on simple assumptions, the age is approximately $14 \pm 2.5$ million years. We used a hydrogen density of 1 atom $/ \mathrm{cm}^{3}$ to calculate its kinetic energy and got $1.9 \pm 0.7 \square 10^{43} \mathrm{~J}$. In the section below, we discuss the implications of the size, expansion velocity, energy, and age estimates.

Table 1. The information of GSH054-00+003

\begin{tabular}{l||c||c}
\hline \hline & Calculated/Observed & Error \\
\hline Location ( RA, Dec) & $292.75^{\circ},+18.25^{\circ}$ & $\sim 0.25^{\circ}$ \\
\hline Galactic Location $(l, b)$ & $53.7^{\circ},-0.1^{\circ}$ & $\sim 0.25^{\circ}$ \\
\hline Mean angular diameter & $1.4^{\circ}$ & $\sim 0.1^{\circ}$ \\
\hline Size & $245 \mathrm{pc}$ & $\pm 10 \mathrm{pc}$ \\
\hline Central velocity & $2 \mathrm{~km} / \mathrm{s}$ & $\pm 1 \mathrm{~km} / \mathrm{s}$ \\
\hline Distance & $10.04 \mathrm{kps}$ & $\pm 0.4 \mathrm{kpc}$ \\
\hline Expansion Velocity & $8.6 \mathrm{~km} / \mathrm{s}$ & $\pm 1.5 \mathrm{~km} / \mathrm{s}$ \\
\hline Age & $14 \mathrm{million}$ years & $+2.5 \mathrm{million}$ ygars \\
\hline Energy $\left(\right.$ density $\left.\mathrm{n}_{\mathcal{H}}=1 / \mathrm{cm}^{3}\right)$ & $1.9 \times 10^{43} \mathrm{~J}$ & $\pm n .7 \times 10^{48} \mathrm{~J}$ \\
\hline Mass(density $\left.\mathrm{n}_{\mathrm{H}}=1 / \mathrm{cm}^{3}\right)$ & $2.7 \square 10^{5} \mathrm{M}_{\text {sun }}$ & $\pm 0.1 \square 10^{5} \mathrm{M}_{\text {sun }}$ \\
\hline \hline
\end{tabular}

\section{Discussion:}

It is certain that the age we calculate is an over estimate, because the shell was expanding faster before. In addition, it is likely this shell is the result of multiple supernovae from a cluster of stars, which exploded at slightly different times and locations. This possibility is discussed further below. However, it is certain that the shell is old. In online archival x-ray images of the shell (McGlynn et al., 1996), the absence of x-ray emission inside the shell means that it is old and cold. The slow expansion velocity further supports that the shell has been expanding for a long time. 
Computer models of supernova evolution suggest that an average old shell with $\mathrm{n}_{\mathrm{H}}=1 \mathrm{atom} / \mathrm{cm}^{3}$ that is expanding at $\sim 10 \mathrm{~km} / \mathrm{s}$ has a kinetic energy of about 3-4 x 10 $0^{42} \mathrm{~J}$ (Spitzer 1978; Chevalier 1974; Thornton, 1998). Thornton's computer models suggest that a single shell expanding into material of number density 1 atom $/ \mathrm{cm}^{3}\left(\mathrm{n}_{\mathrm{H}}=1\right)$ and currently expanding at $9 \mathrm{~km} / \mathrm{s}$ would be around 1.7 million years old, around $70 \mathrm{pc}$ in radius and have kinetic energy $4 \square 10^{42} \mathrm{~J}$. We used this model as a reference. GSH054-00+003 is bigger and contains more energy than this model, so we suspect it to be a cluster of supernovae. We first used the radius to estimate how many supernovae formed GSH054-00+003. Assuming it is a perfect sphere, we calculated its volume to be $2.5 \square 10^{6} \pi \mathrm{pc}^{3}$. For a supernova that expands into material with $\mathrm{n}_{\mathrm{H}}=1$, the volume will be $460,000 \pi \mathrm{pc}^{3}$. This volume suggests that GSH054-00+003 was made by about 6 supernovae. Then, we compared the kinetic energies. Dividing GSH054-00+003's energy by the model's energy, we get around 5 to 7 supernovae. If we assume the density to be higher, there will be more mass swept up and therefore more energy was needed to form GSH054-00+003. This also means the shell needed more supernovae to form. For a computer model with $\mathrm{n}_{\mathrm{H}}=10$ (Thornton, 1998), a shell expanding at $9 \mathrm{~km} / \mathrm{s}$ will be 6.6 million years old, $30 \mathrm{pc}$ in radius and have kinetic energy around $2.5 \square 10^{42} \mathrm{~J}$. This suggests GSH054-00+003 is made up by 70 supernovae if comparing with radius, and 60 to 100 supernovae if comparing with the energy. It is unlikely that the shell will expand into material with density less than 1 atom $/ \mathrm{cm}^{3}$, because it is near a spiral arm, which is very dense. To conclude, GSH054-00+003 is an old shell, likely formed by a cluster of supernovae.

\section{Acknowledgements:}

I would like to acknowledge the use of NASA's SkyView facility (http://skyview.gsfc.nasa.gov) located at NASA Goddard Space Flight Center. In addition, I would like to thank Dr. E. J. Korpela and the SETI@home team for access to the SETHI dataset, which uses data acquired at Arecibo Observatory. The Arecibo Observatory is part of the National Astronomy and Ionosphere Center, which is operated by Cornell University under a Cooperative Agreement with the National Science Foundation.

\section{Reference:}

Brand, J. \& Blitz, L. 1993, A\&A, 275, 67

Chevalier, A. Ap.J., 188, 501, 1974

Cordes, J. \& Lazio, J. 2003, arXiv: astro-ph/0301598

Cox, D. P. 2005, ARA\&A, 43, 337

Gooch, R. 1996, Astronomical Data Analysis Software and Systems V, 101, 80

Korpela, E. J., Demorest, P., Heien, E., Heiles, C., \& Werthimer, D. 2002, Seeing Through the

Dust: The Detection of HI and the Exploration of the ISM in Galaxies, 276, 100

Korpela, E. J., Demorest, P., Heien, E., Heiles, C., \& Werthimer, D. 2004, How Does the Galaxy Work?, 315, 97

McGlynn, T., Scollick, K., White, N., SkyView: The Multi-Wavelength Sky on the Internet, McLean, B.J. et al., New Horizons from Multi-Wavelength Sky Surveys, Kluwer Academic Publishers, 1996, IAU Symposium No. 179, p465.

Spitzer, L. 1978. Physical Processes in the ISM

Thornton, K. et al. 1998, ApJ, 500, 95 